\title{
Hyperbolic cosine function in physics
}

\author{
Isabelle Bonnet ${ }^{1,2}$ and Julien Gabelli ${ }^{3}$ \\ ${ }^{1}$ Laboratoire Physico Chimie Curie, Institut Curie, PSL Research University, \\ CNRS UMR 168, 26 rue d'Ulm, 75005, Paris, France \\ 2 Sorbonne Université, 75005, Paris, France \\ 3 Université Paris Saclay, CNRS, Laboratoire de Physique des Solides, 91405 Orsay, \\ France \\ E-mail: julien.gabelli@u-psud.fr
}

\begin{abstract}
A catenary is the shape that an electric cable takes under its own weight if suspended only at its ends between two pylons. A rope hung between two masts is also described with a catenary equation which is based on the hyperbolic cosine function. We propose here to approach the hyperbolic cosine function with two simple experiments using a beaded chain. We first study the static form of a chain whose ends are suspended between two poles, when the distance between the two poles varies. Then, we study the dynamics of the same chain falling from the edge of a table. The simplicity of these two experiments, which can be done at home, makes them appropriate for undergraduate students interested in practicals.
\end{abstract}

Keywords: catenary, chainette, physics, hyperbolic cosine

\section{Introduction}

A catenary is a two-dimensional curve that describes the shape of an homogeneous flexible chain suspended by its ends so that it is sagging only subject to gravity. In the early 17 th century, Galileo was the first to address the mathematical question of the shape of a chain hanging under gravity; he wrongly claimed that the shape would fit a parabola [1,2]. A few years later, in 1690, Jacob Bernoulli launched a challenge to find the mathematical description of a suspended chain shape. One year after, the curve equation was obtained by Leibniz et al: they called it 'the catenary equation' [3-5]. It corresponds to the hyperbolic cosine function cosh, and is also known as a 'chainette' in textbooks. This mathematical function can be expressed in terms of exponential functions as $\cosh (x)=\frac{\mathrm{e}^{x}+\mathrm{e}^{-x}}{2}$. 
In this paper, we propose to study the hyperbolic cosine function with two simple experiments that can be performed at home with everyday life objects: a chain, a smartphone camera and a computer. Both experiments deal with classical physical problems: the first experiment is static (the analysis of the form taken by a hanging chain [6, 7]) while the second one is dynamics (the motion of a chain sliding from a horizontal surface [8]). They are thus appropriate for undergraduate students.

\section{The shape of the chain}

\subsection{Theoretical description}

We consider a chain of length $L$ and mass $M$ - which is uniform-and we denote $\mu=\frac{M}{L}$ its mass per unit length. We use Cartesian coordinates $(x, z)$, with $x$ the horizontal axis and $z$ the vertical axis (see figure 1 for notations). The two extremities of the chain are placed at $x=0$ and $x=h$, so that $z(0)=z(h)$. Moreover, we impose the origin of the $z$-axis such as $z(0)=0$. Applying the equilibrium condition on a chain's element of length $\mathrm{d} l$ in the $x$ and $z$ directions leads to

$$
\mid \begin{aligned}
& \mathrm{d}(T \cdot \cos \alpha)=0, \\
& \mathrm{~d}(T \cdot \sin \alpha)-\mu g \mathrm{~d} l=0,
\end{aligned}
$$

where $\mathrm{d} l=\sqrt{\mathrm{d} x^{2}+\mathrm{d} z^{2}}=\mathrm{d} x \sqrt{1+\left(\frac{\mathrm{d} z}{\mathrm{~d} x}\right)^{2}}$ and $\alpha$ the angle the segment $\mathrm{d} l$ makes with the $x$-axis. We then deduce

$$
\mid \begin{aligned}
& T(x) \cdot \cos \alpha(x)=A \\
& \mathrm{~d}(T \cdot \cos \alpha \cdot \tan \alpha)=\mu g \sqrt{1+\left(\frac{\mathrm{d} z}{\mathrm{~d} x}\right)^{2}} \mathrm{~d} x
\end{aligned}
$$

with $A$ a constant, which depends on the distance $h$ between the two chain's ends: we will denote it $A(h)$, and $\frac{\mathrm{d} z}{\mathrm{~d} x}=\tan \alpha$. Knowing that $\frac{\mathrm{d}}{\mathrm{d} u} \operatorname{argsh}(u)=\frac{1}{\sqrt{1+u^{2}}}$, we obtain, after integration, the following differential equation:

$$
\arg \operatorname{sh}\left(\frac{\mathrm{d} z}{\mathrm{~d} x}\right)-\arg \operatorname{sh}\left(\left.\frac{\mathrm{d} z}{\mathrm{~d} x}\right|_{x=h / 2}\right)=\frac{\mu g}{A}\left(x-\frac{h}{2}\right)
$$

Because of the symmetry, $\arg \operatorname{sh}\left(\left.\frac{\mathrm{d} z}{\mathrm{~d} x}\right|_{x=h / 2}\right)=0$, and we get $\frac{\mathrm{d} z}{\mathrm{~d} x}=\sinh \left(\frac{\mu g}{A}\left(x-\frac{h}{2}\right)\right)$. Knowing that $\cos \alpha=\frac{\mathrm{d} x}{\mathrm{~d} l}=\frac{1}{\sqrt{1+\frac{\mathrm{dz}}{\mathrm{d} x}}}$, we finally deduce

$$
\mid \begin{aligned}
& z(x)=\xi \cosh \left(\frac{x-h / 2}{\xi}\right)-\xi \cosh \left(\frac{h}{2 \xi}\right), \\
& T(x)=\frac{A}{\cos \alpha}=A \cosh \left(\frac{x-h / 2}{\xi}\right),
\end{aligned}
$$

with $\xi(h)=\frac{A(h)}{\mu g}$. 


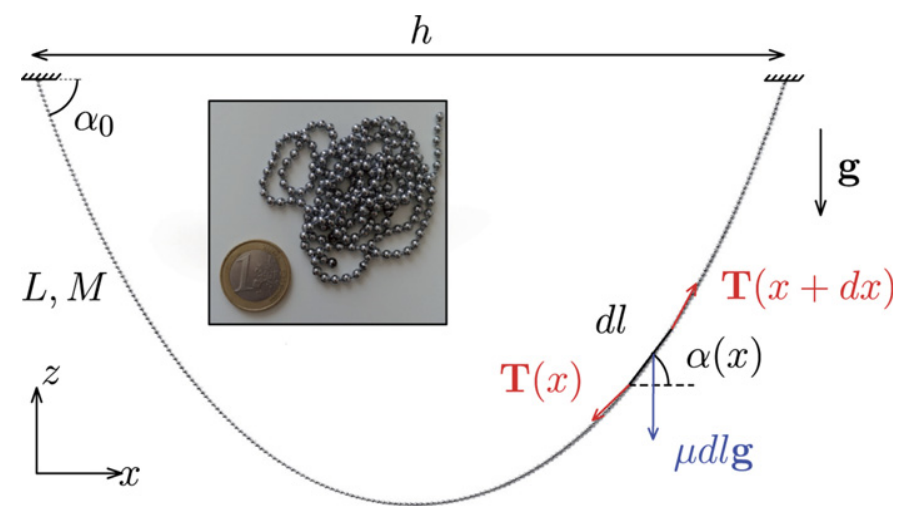

Figure 1. Picture of the beaded chain used in this paper, suspended only by its ends, with the notations used for the derivation of the catenary equation. The two extremities of the chain (of length $L$ and mass $M$ ) are separated by a distance $h$. Vector quantities are represented by bold letters and we use the Cartesian coordinate plane $(x, z): g$ is the acceleration produced by gravity at the Earth's surface. A segment of chain, of length $\mathrm{d} l$, at coordinates $(x, z)$ is subject to the tension of the chain $\mathbf{T}(x)$ at point $x$, to the tension of the chain $\mathbf{T}(x+\mathrm{d} x)$ exerted at the point $x+\mathrm{d} x$ and to its weight $\mu \mathrm{d} l g$ with $\mu$ the linear mass density of the chain. This segment makes an angle $\alpha(x)$ with the $x$-axis. Inset: Picture of the chain laid on a table to distinguish the individual beads that make it up.

\subsection{Chain's characteristic}

We used a beaded chain of length $L=952 \mathrm{~mm}$ and mass $M=21 \mathrm{~g}$ : its mass per unit length is thus $\mu=\frac{M}{L}=22 \mathrm{~g} \cdot \mathrm{m}^{-1}$. The chain is composed of $N_{\text {beads }}=221$ beads and is suspended between its two ends separated by a distance $h$ varying from 30 to $80 \mathrm{~cm}$ (figure 1). For each distance $h$, we took a picture of the chain with a smartphone fixed to a camera tripod equipped with a bubble level to ensure horizontality: we checked that the camera's optical axis was perpendicular to the plane of the chain. We measured the $(x(n), z(n))$ coordinates of points of the chain by clicking manually on the $n \in[[0 ; 221]]$ beads with the multi-points tool in ImageJ [9]. We then reconstructed the shape of the chain point by point, as shown on figure 2, which displays the measured profiles $z(x)$ for the different values of $h$.

We first measured the distance $a(n)$ between two successive beads $a(n)=\sqrt{(x(n+1)-x(n))^{2}+(z(n+1)-z(n))^{2}}$ and found $a=\langle a(n)\rangle=4.4 \pm 0.1 \mathrm{~mm}$ (inset of the figure 2), in very good agreement with the expected value $a=\frac{L}{N_{\text {beads }}-1}=4.3 \mathrm{~mm}$.

\subsection{Experimental description of the chain curve}

To compare the data with the catenary equation (equation (4)), we fitted our experimental data (figure 2) with the theoretical expectation $z(x)=\xi \cosh \left(\frac{x-x_{0}}{\xi}\right)-\xi \cosh \frac{x_{0}}{\xi}$ leaving $x_{0}(h)$ and $\xi(h)$ as free parameters. The graph of $x_{0}$ as a function of $h$ displays a linear behavior and we found $\frac{x_{0}}{h}=0.511 \pm 0.002$, as expected (figure 3(a)). The parameter $\xi(h)$ can be related to the total chain's length $L$ since $L=\int_{0}^{h} \sqrt{1+\frac{\mathrm{d} z}{\mathrm{~d} x}} \mathrm{~d} x=2 \xi \sinh \left(\frac{h}{2 \xi(h)}\right)$. We numerically solved this nonlinear equation, which allows us to deduce a theoretical expectation of $\xi(h)$, as shown in figures 3(b) and 4. Moreover, the inset of figure 3(b) displays the graph $\sinh \left(\frac{h}{2 \xi(h)}\right)$ as a function of $\frac{L}{2 \xi(h)}$; a linear fit gives a slope of $0.989 \pm 0.004$, in very good agreement with the theory. 


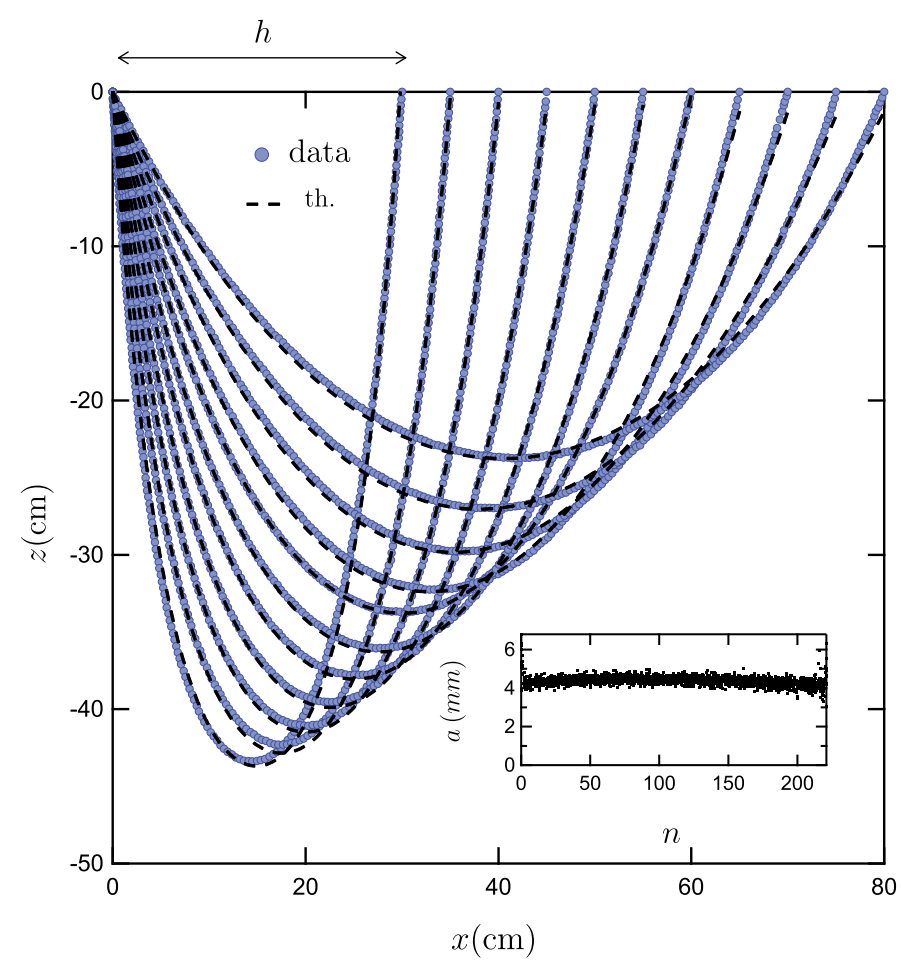

Figure 2. Shape of the suspended chain when the distance $h$ between its two ends is varying: $h=30,35,40,45,50,55,60,65,70,75$ and $80 \mathrm{~cm}$. The blue dots are obtained by manually clicking on the beads on the picture of the chain. These data points are fitted with the theoretical curve of the catenary equation $z(x)=\xi \cosh \left(\frac{x-h / 2}{\xi}\right)-$ $\xi \cosh \left(\frac{h}{2 \xi}\right)$ (black dashed lines, $\xi$ and $x_{0}$ being free parameters). Inset: Experimental determination of the distance $a$ between two successive beads; $n$ is the bead index of the chain $(n=0 \ldots 221)$; we find $a \simeq 4.4 \pm 0.1 \mathrm{~mm}$. The pointing error is estimated to be $0.5 \mathrm{~mm}$, except at the chain's extremities where the pointing is delicate because of the fixing.

\subsection{Tension at the chain's extremities}

Let us denote $T(0)=T(h) \equiv T_{0}$ and $|\alpha(0)|=|\alpha(h)| \equiv \alpha_{0}$ the tension and the angle at the chain's extremities which both depend on $h$. The equilibrium condition for the whole chain is written as $2 T_{0} \sin \alpha_{0}=2 T_{0} \cos \alpha_{0} \tan \alpha_{0}=M g$. The angle $\alpha_{0}$ is deduced from the ended points of the chain: $\alpha_{0}=\frac{1}{2}\left(\arctan \frac{z\left(N_{\text {beads }}\right)-z\left(N_{\text {beads }}-1\right)}{x\left(N_{\text {beads }}\right)-x\left(N_{\text {beads }}-1\right)}-\arctan \frac{z_{0}-z_{1}}{x_{0}-x_{1}}\right)$. Since $T_{0}=\frac{M g}{2 \sin \alpha_{0}}$ and $\sin \alpha=\frac{\mathrm{d} z}{\mathrm{~d} l}=\frac{\mathrm{d} z / \mathrm{d} x}{\sqrt{1+(\mathrm{d} z / \mathrm{d} x)^{2}}}=\frac{\sinh \left(\frac{x-h / 2}{\xi}\right)}{\cosh \left(\frac{x-h / 2}{\xi}\right)}$, we obtain $T_{0}=\frac{M g}{2 \tanh \left(\frac{h}{2 \xi(h)}\right)}$. Figure 4 shows the graph of $T_{0}(h)$ where the gravity at the Earth's surface was assumed to be $9.8 \mathrm{~m} \cdot \mathrm{s}^{-2}$. Note that $\lim _{h \rightarrow L} T_{0}(h)=+\infty$ : a suspended horizontal cable would imply an infinite tension on the posts. It is therefore impossible to have a perfectly horizontal catenary: that is why droppers are used to connect the catenary with a contact wire which is horizontal in the electrical overhead lines. 

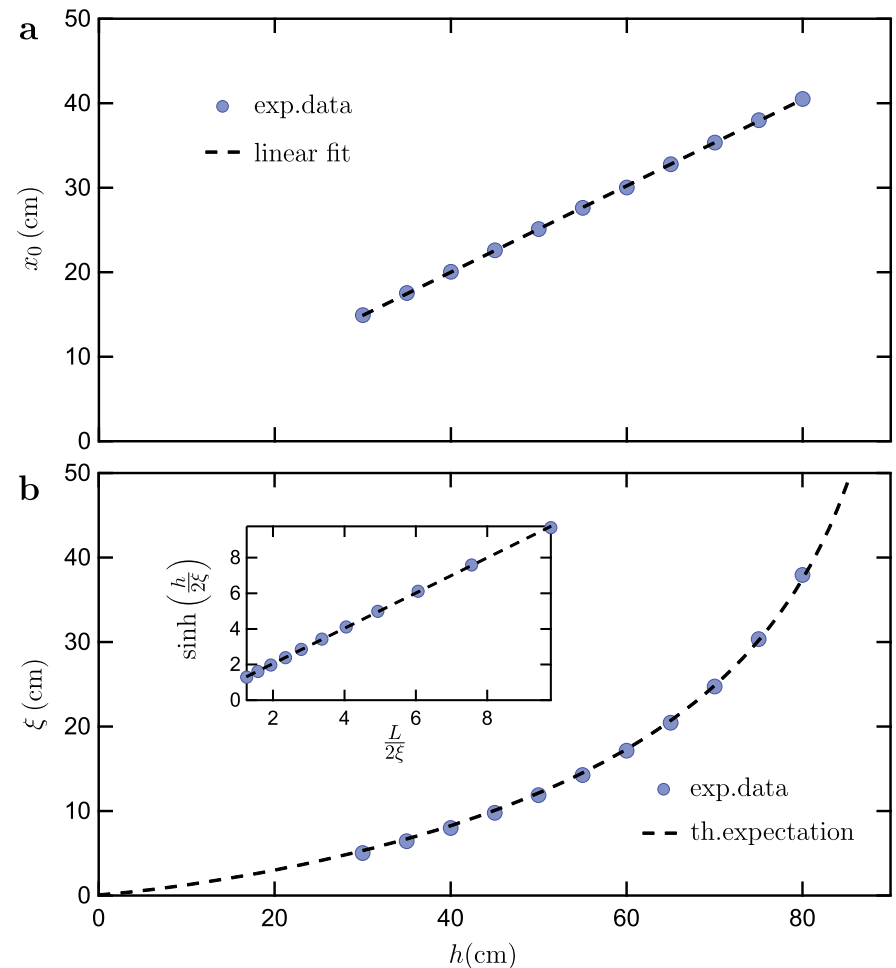

Figure 3. (a) Graph of the free parameter $x_{0}$ as function of the distance $h$ between the fixed point displays a linear behavior with a slope of $1 / 2$, as expected with equation (4). (b) The fitting parameter $\xi$ as a function of $h$ is in agreement with the theoretical expectation we numerically obtained.

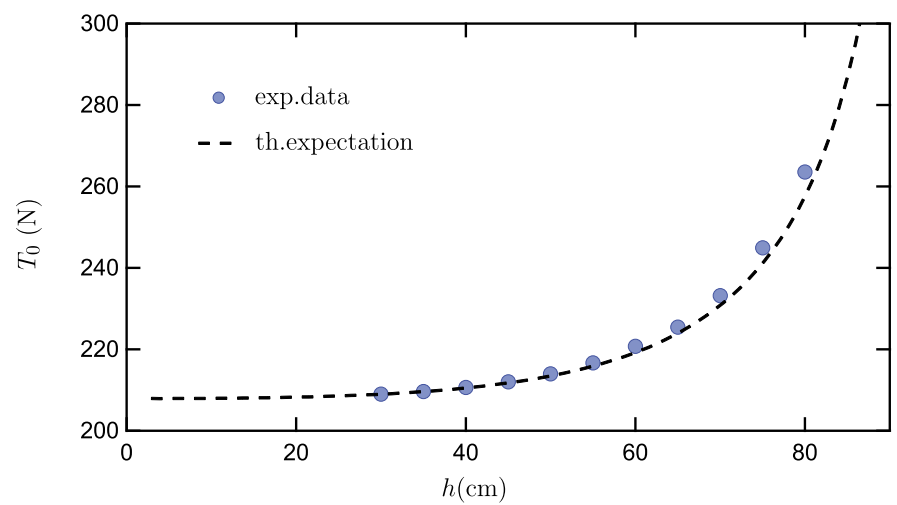

Figure 4. Graph of the chain tension at its extremities. Experimental points (blue circle) estimated with the formula $\frac{M g}{2 \sin \alpha_{0}(h)}$, compared to theoretical values $\frac{M g \xi(h) \cosh \frac{x_{0}}{\xi(h)}}{L}$ (dashed black line). 


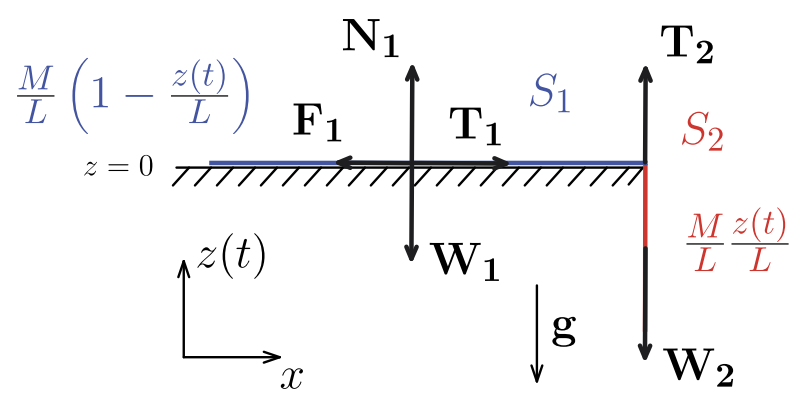

Figure 5. Schematic of the forces acting on a chain falling from the edge of a table at a time $t>0$. We denote $z(t)$ the length of the chain hanging out of the table, the surface of the table being defined by $z=0$. The reference time $t=0$ corresponds to the beginning of the fall. We split the chain into two subsystems. The first one, $S_{1}$ (in blue), corresponds to the part of the chain in contact with the table at time $t$, and has a length of $L-z(t)$. Four forces act on $S_{1}$ : its weight $\mathbf{W}_{\mathbf{1}}$, the two components of the reaction $\left(\mathbf{N}_{\mathbf{1}}, \mathbf{F}_{\mathbf{1}}\right)$ and the tension $\mathbf{T}_{\mathbf{1}}$ exerted by the other part of the chain. The second subsystem is $\mathrm{S}_{2}$ (in red) and is the part of the chain of length $z(t)$ that is hanging over. $S_{2}$ is subject to two forces: the weight $\mathbf{W}_{\mathbf{2}}$ and the tension $\mathbf{T}_{\mathbf{2}}$. The chain is supposed to be inextensible so that $\left\|\mathbf{T}_{1}\right\|=\left\|\mathbf{T}_{\mathbf{2}}\right\|$.

\section{Fall of the chain from the edge of a table}

In this second part, we approach the mathematical function cosh differently by studying another classical physics problem: the dynamics of a chain falling from the edge of a table.

\subsection{Theoretical description}

We consider a similar beaded chain, but with different characteristics: it is now composed of $N_{\text {beads }}=229$ beads so that its length is now $L=982 \mathrm{~mm}$ and its mass is $M=22 \mathrm{~g}$. The chain is fully stretched and lying on a table so that its direction is perpendicular to the edge of the table.

One part of the chain is horizontal and lying on the table while the other part, of length $z(t)$, is hanging vertically off of the table (figure 5). We split the chain into two subsystems: $S_{1}$ corresponds to the part of the chain in contact with the table and has a length of $L-z(t)$. The second subsystem is $\mathrm{S}_{2}$ and is the part of the chain (of length $z(t)$ ) that is hanging off.

The chain will start to move as soon as its hanging part $z>z_{0}$, where $z_{0}$ is a threshold length, and corresponds to the minimal length of the chain required to trigger the fall of the entire chain by gravity. The start of the motion sets the reference time $t=0$. We measured a threshold length corresponding to 35 beads, or $z_{0}=154 \mathrm{~mm}$.

The amplitudes of the forces acting on $\mathrm{S}_{1}$ are (see figure 5 for notations):

$$
\mid \begin{array}{ll}
W_{1}=-M g\left(1-\frac{z}{L}\right) & \begin{array}{l}
\text { its weight, } \\
\text { the normal reaction of the table, } \\
N_{1}=-W_{1} \\
F_{1}=-k N_{1}
\end{array} \\
T_{1} & \begin{array}{l}
\text { the friction exerted by the table, } \\
\text { the tension exerted by } \mathrm{S}_{2}
\end{array}
\end{array}
$$

with $k$ the coefficient of friction [10]. For $t \geqslant 0$, the chain is moving so that $k=\mu_{\mathrm{k}}<\mu_{\mathrm{s}}$ where $\mu_{\mathrm{k}}$ and $\mu_{\mathrm{s}}=\left|\frac{F_{1}}{N_{1}}\right|$ are the coefficients of kinetic and static friction, respectively. The latter can be 


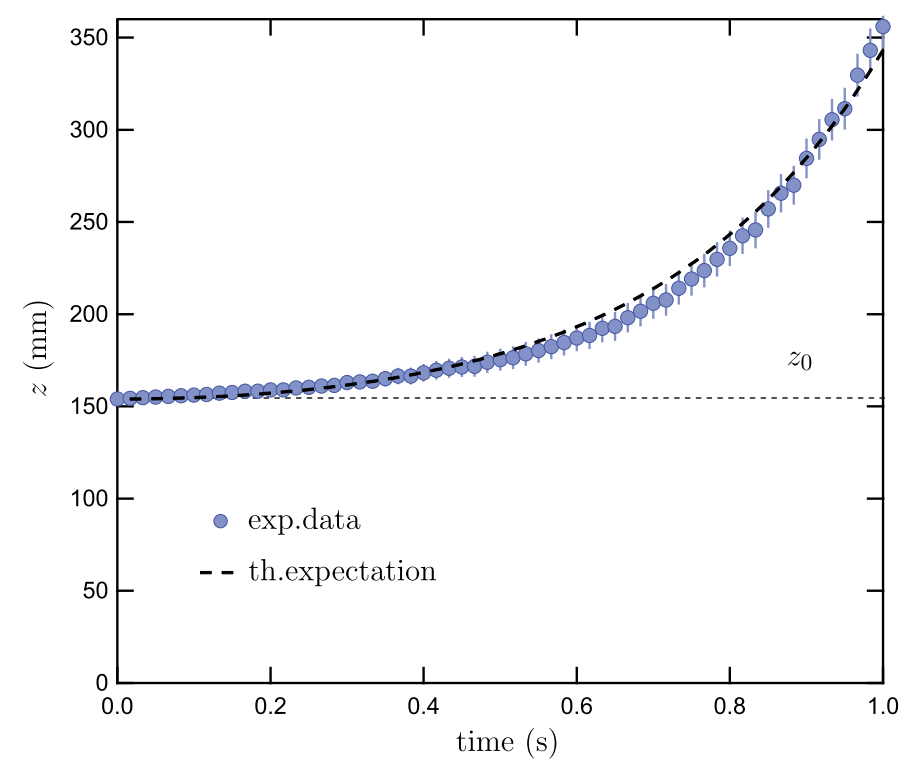

Figure 6. Chain falling from a table. Comparison between experimental points (blue circles) of $z(t)$ obtained by manually tracking the lowest bead of the chain, and theoretical expectation (dashed black line) during the fall of the chain (equation (8)).

estimated with $z_{0}$. The equilibrium before motion $(t<0)$ gives $N_{1}+W_{1}=0$ and $F_{1}+T_{1}=0$ for $\mathrm{S}_{1}$ and $W_{2}+T_{2}=0$ for $\mathrm{S}_{2}$. Moreover, assuming the chain is inelastic, we have $\left|T_{1}\right|=\left|T_{2}\right|$. Finally, $\mu_{\mathrm{s}}=\left|\frac{F_{1}}{N_{1}}\right|=\frac{W_{2}}{W_{1}}=\frac{z_{0}}{L-z_{0}} \simeq 0.186$.

Even if the system is not conservative, it is convenient to use the work energy theorem on the whole system:

$$
\frac{\mathrm{d} \mathcal{E}}{\mathrm{d} t}=\mathcal{P}
$$

where $\mathcal{E}$ is the total energy including kinetic energy $K=\frac{M}{2}\left(\frac{\mathrm{d} z}{\mathrm{~d} t}\right)^{2}$ and the potential energy $U=\int-\mu g z \mathrm{~d} z=-\frac{M g}{2 L} z^{2}$ with the assumption $U(z=0)=0$. The dissipated power due to friction on the table is $\mathcal{P}=F_{1} \frac{\mathrm{d} z}{\mathrm{~d} t}=-M g \mu_{\mathrm{k}}\left(1-\frac{z}{L}\right) \frac{\mathrm{d} z}{\mathrm{~d} t}$. Finally, we get a second-order differential equation:

$$
\frac{\mathrm{d}^{2} z}{\mathrm{~d} t^{2}}-\frac{z}{\tau^{2}}=-g \mu_{\mathrm{k}}
$$

where $\tau=\sqrt{\frac{L}{g\left(1+\mu_{\mathrm{k}}\right)}}$ is the characteristic time of the fall. Since the chain falls without initial velocity and having $z(0)=z_{0}$, we obtain

$$
z(t)=\left(z_{0}-\frac{\mu_{\mathrm{k}}}{1+\mu_{\mathrm{k}}} L\right) \cosh \left(\frac{t}{\tau}\right)+\frac{\mu_{\mathrm{k}}}{1+\mu_{\mathrm{k}}} L
$$

where we have assumed that $z(0)=z_{0}+\varepsilon$, with $\varepsilon$ an infinitely small positive quantity, so that the motion is already initiated at time $t=0$. 


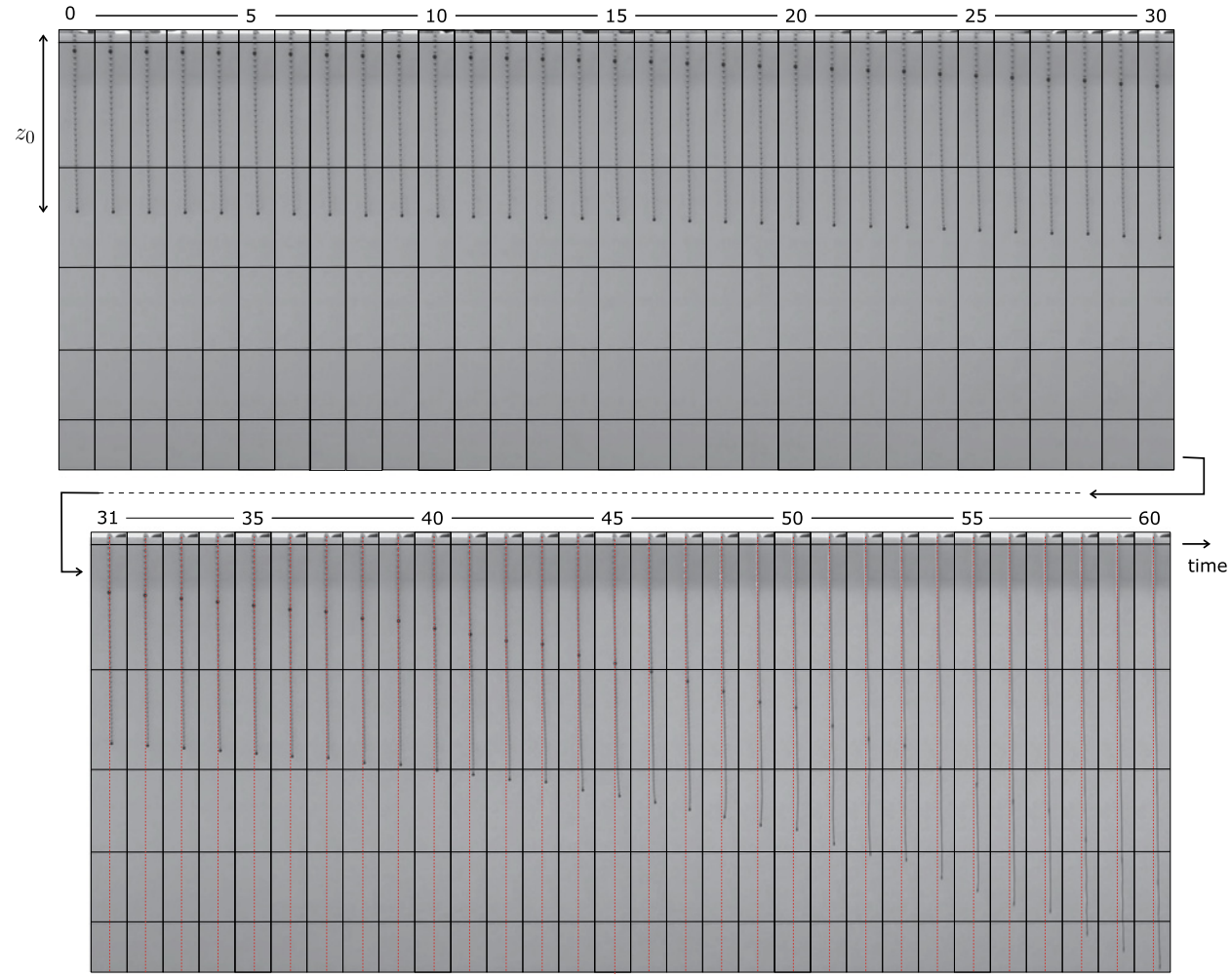

Figure 7. Time-lapse of the chain falling. The time is indicated in frame; the total fall lasts one second. For sake of visibility, we painted two beads in black. Starting from frame 31 , we have indicated the vertical axis with dashed red lines to emphasize the fact that the chain is snaking while falling.

\subsection{Experimental data}

We manually tracked the lowest bead of the chain (which was painted in black). The theoretical fitting of the graph $z=f(t)$ gave $\mu_{\mathrm{k}}=0.1672 \pm 0.0001$ (figure 6) and we then deduced $\tau=0.293 \pm 0.002 \mathrm{~s}$, where we have used a value of $9.8 \mathrm{~m} \cdot \mathrm{s}^{-2}$ for $g$. As expected, note that $\mu_{\mathrm{k}}<\mu_{\mathrm{s}}$. On figure 6 , we see a slight discrepancy between the experimental data and the theoretical expectation. This is due to experimental uncertainty but also to the theoretical modeling which assumed a constant coefficient of kinetic friction. The coordinates measurement is actually complicated as soon as the chain's velocity exceeds the frame rate acquisition $(\Delta t=1 / 60 \mathrm{~s})$ : this is the case when $\frac{\mathrm{d} z}{\mathrm{~d} t}>\frac{a}{\Delta t}=\simeq 0.3 \mathrm{~m} \cdot \mathrm{s}^{-1}$ (frames 30-60 in figure 7). We also noticed that the chain snakes horizontally starting from frame 31 , maybe due to the chamfer of the table edge.

\section{Conclusions}

Through this study, we have illustrated the importance of the hyperbolic cosine function in simple mechanics. The experiments carried out deal with static and dynamic studies of the same system: a chain characterized by its mass $M$ and its length $L$. They have allowed us to characterize a shape by a characteristic length $\xi=\frac{T_{0} L \cos \alpha_{0}}{M g}$ and a movement by a 
characteristic time $\tau=\sqrt{\frac{L}{g\left(1+\mu_{\mathrm{k}}\right)}}$. In the first experiment, the chain is maintained by posts at an angle $\alpha_{0}$ and a tension $T_{0}$ while in the second it falls by rubbing on a table with a coefficient of kinetic friction $\mu_{\mathrm{k}}$. In both cases the chain is subjected to gravity $g$ so that the acceleration $\frac{\xi}{\tau^{2}}$ depends on the characteristics of the chain (its mass $\left.M\right)$, on the boundary conditions $\left(T_{0}\right.$ and $\left.\alpha_{0}\right)$ and on the support's characteristics $\left(\mu_{\mathrm{k}}\right): \frac{\xi}{\tau^{2}}=\frac{\left(1+\mu_{\mathrm{k}}\right) T_{0} \cos \alpha_{0}}{M}$. This relates the universal character of gravity in both experiments: static or dynamical.

The two experiments presented above can be easily conducted at home since they do not require any specialized equipment: they just require a camera, a chain, a measuring tape, a kitchen weighting scale and a personal computer. Analyses can be achieved thanks to free software: ImageJ for the tracking, and Python for the numerical solving of equations. This study is thus appropriate for undergraduate students.

\section{References}

[1] Galilei G 1914 Dialogues Concerning Two New Sciences trans. H Crew and A de Salvio (New York: Macmillan)

[2] Galilei G 1960 Dialogue Concerning the Two Chief World Systems-Ptolemaic \& Copernican (Berkeley, CA: University of California Press)

[3] Leibniz 1691 De Linea in Quam Flexile se Pondere Propio Curvat, Ejusque Usu Insigni ad Inveniendas Quotcunque Medias Proportionales et Logarithmos (Leipzig: Acta Eruditorum) pp 243-7

[4] Huygens 1691 Dynastae in züchelem Solutio Ejusdemproblematis (Leipzig: Acta Eruditorum) pp 281-2

[5] Bernoulli J 1691 Solutio Problematis Funicularis, Exhibita a Johanne Bernoulli (Leipzig: Acta Eruditorum) pp 274-6

[6] Nedev S 2000 The catenary-an ancient problem on the computer screen Eur. J. Phys. 21, p 451-457

[7] Behroozi F 2018 In praise of the catenary Phys. Teach. 56, p 214-217

[8] Behroozi F 1997 The sliding chain problem with and without friction: a universal solution Eur. J. Phys. 18, p 15-17

[9] Schindelin J, Arganda-Carreras I and Frise E et al (2020) Fiji: an open-source platform for biological-image analysis, Nature methods 9(7) 676-682 PMID 22743772

[10] Feynman R P 1963 The Feynman Lectures on Physics Volume 1: Mainly mechanics, Radiation, and Heat (Reading, MA: Addison-Wesley) 